\title{
Severity and outcome of cystic lung disease in women with tuberous sclerosis complex
}

\author{
Angelo M. Taveira-DaSilva ${ }^{1}$, Amanda M. Jones ${ }^{1}$, Patricia Julien-Williams ${ }^{1}$, \\ Jianhua Yao ${ }^{2}$, Mario Stylianou ${ }^{3}$ and Joel Moss ${ }^{1}$
}

Affiliations: ${ }^{1}$ Cardiovascular and Pulmonary Branch, National Institutes of Health Heart, Lung and Blood Institute, National Institutes of Health, Bethesda, MD, USA. ${ }^{2}$ Radiology and Imaging Sciences Department, National Institutes of Health, Bethesda, MD, USA. ${ }^{3}$ Office of Biostatistics Research, National Institutes of Health Heart, Lung and Blood Institute, National Institutes of Health, Bethesda, MD, USA.

Correspondence: Angelo M. Taveira-DaSilva, Cardiovascular and Pulmonary Branch, NHLBI, NIH, Building 10, Room 6D03, MSC 1590, Bethesda, Maryland 20892-1590, USA. E-mail: dasilvaaAnhlbi.nih.gov

ABSTRACT What are the clinical features, severity, and rate of progression of lung disease in women with tuberous sclerosis and lymphangioleiomyomatosis (LAM) and how do they differ from patients with sporadic LAM?

Data from 94 tuberous sclerosis/LAM and 460 sporadic LAM women were compared. 40 tuberous sclerosis/LAM and 40 sporadic LAM patients were age- and lung function-matched, and changes in volume occupied by cysts (cyst score) and pulmonary function occurring over 6.5 years were evaluated.

Tuberous sclerosis/LAM patients had better lung function than sporadic LAM patients, but no difference was observed from sporadic LAM patients in yearly rates of change in forced expiratory volume in $1 \mathrm{~s}(-1.9 \pm 2.7$ versus $-1.9 \pm 1.9 \%$ predicted; $\mathrm{p}=0.302)$, diffusing capacity of the lung for $\mathrm{CO}(-2.1 \pm 2.8$ versus $-1.9 \pm 2.7 \%$ predicted; $\mathrm{p}=0.282)$ or cyst scores $(+1.0 \pm 1.3$ versus $+1.4 \pm 1.7 \%, \mathrm{p}=0.213)$. However, the proportion of patients with abnormal lung function and higher rates of FEV1 decline was greater in sporadic LAM. Some young tuberous sclerosis/LAM patients (mean age $25.7 \pm 3$ years) progressed rapidly from minimal to severe lung disease.

Tuberous sclerosis/LAM patients may experience abrupt declines in lung function. Consequently, women with tuberous sclerosis found to have lung cysts should undergo periodic functional and radiological testing to follow disease progression and determine need for therapy.

@ERSpublications

While the natural history is variable, some women with TSC and LAM may quickly progress to disabling respiratory disease http://ow.ly/Dywa7

This article has supplementary material available from erj.ersjournals.com

Received: May 132014 | Accepted: Oct 222014

Support statement: This study was supported by the Intramural Research Program, National Institutes of Health, National Heart, Lung, and Blood Institute.

Conflict of interest: None declared

Copyright @ERS 2015 


\section{Introduction}

Lymphangioleiomyomatosis (LAM), a multisystem disease affecting predominantly women, is characterised by cystic lung destruction, abdominal angiomyolipomas, lymphatic tumours and chylous effusions [1-3]. The pathological features of LAM are caused by proliferation of a neoplastic smooth muscle-like LAM cell that has characteristics of both smooth muscle cells and melanocytes [1, 4]. An inherited form of LAM occurs in patients with tuberous sclerosis complex (TSC), an autosomal dominant disorder which occurs in one of 6000 live births and is caused by mutations in the tuberous sclerosis complex 1 (TSC1) or 2 (TSC2) genes [5, 6]. A second form, sporadic LAM, is an uncommon disease affecting 3-5 per million women in the general population, and is caused by mutations of the TSC2 gene [5, 7-9]. Loss of heterozygosity of TSC2 has been reported in LAM lesions from lung and kidney angiomyolipomas and LAM cells isolated from lung, blood and chyle [5, 8, 10-12]. The metastatic nature of LAM is supported by the presence of identical mutations in lung and kidney lesions and evidence of de novo invasion of transplanted donor's lungs by the recipient LAM cells, suggesting migration from the kidney, lymphatic system or uterus, to the lungs [13-15].

The prevalence of clinically significant LAM in TSC was thought to be low, ranging from 0.6 to $2.3 \%$ of TSC patients [16-19]. Subsequent cross-sectional studies however, showed that the prevalence of lung cysts in women with TSC ranged from 26 to $49 \%$ and that the extent of cystic disease increases with age [2025]. Clinically significant cystic disease is almost exclusively seen in women.

Although the clinical and physiological features of sporadic LAM have been described, there are large gaps in our knowledge of the natural history of TSC-LAM, especially whether the prognosis of TSC-LAM is similar to that of sporadic LAM and whether a few lung cysts on a computed tomography scan of a woman with TSC and normal lung function may progress to clinically significant lung disease $[1,26,27]$. We also wanted to determine whether the rates of change in lung function and lung volume occupied by cysts [28] in patients with TSC-LAM were different from those with sporadic LAM. To answer these questions, we reviewed the clinical and physiological characteristics and high-resolution computed tomography (HRCT) scans, of TSC-LAM patients seen at the National Institutes of Health (NIH) Clinical Research Center (Bethesda, MD, USA) over a period of 16 years and compared their features with those of patients with sporadic LAM.

\section{Material and methods}

\section{Patient population}

Between 1996 and 2012, 637 women were referred to NIH for participation in a natural history and pathogenesis protocol (NIH Heart, Lung and Blood Institute (NHLBI) Protocol 95-H-0186), and a lung disease screening protocol (NHLBI Protocol 82-H-0032) which were approved by the Institutional Review Board of the NHLBI. Patients were either self-referred or referred through individual physicians. All subjects gave written informed consent before enrolment. Of the 637 patients, 554 had LAM. History and physical examination, laboratory tests and pulmonary function tests were obtained at the time of each visit. Cardiopulmonary exercise tests, CT scans of the thorax and abdomen and magnetic resonance imaging of the brain were obtained once a year. Data from some of these patients have been reported previously $[1,26,29]$.

\section{Pulmonary function testing}

Lung function was measured (MasterScreen PFT; CareFusion Corporation, San Diego, CA, USA) according to standard methods $[30,31]$.

\section{Cardiopulmonary exercise testing}

Patients exercised on a bicycle ergometer and a computerised metabolic cart (Vmax 229 Cardiopulmonary Exercise System; CareFusion Corporation, San Diego, CA, USA) using incremental protocols [32].

\section{Radiological methods}

40 TSC-LAM patients were selected based on the suitability of CT scan studies for a computer analysis that estimates the volume of lung occupied by cysts (cyst scores) and lung texture changes in areas adjacent to cysts [28]. Once this was accomplished, from the sporadic LAM cohort, we selected patients who not only had suitable CT scans for analysis but also matched each TSC-LAM patient by age, lung function and follow-up time. Then, we estimated their cyst scores, rate of progression of cystic lesions and rates of decline in lung function.

\section{Statistical methods}

For comparisons of demographic data, lung function and exercise tests between TSC-LAM and sporadic LAM patients, a t-test was employed. The data set contained clinical data and multiple pulmonary 
function and exercise measurements for most patients. The yearly rate of change (slope) was calculated from a linear regression for pulmonary function and exercise data [26], and an adjusted analysis was then performed using mixed effects models. All available data were used to compare TSC-LAM and sporadic LAM patients using a t-test for continuous variables and a Chi-squared test for categorical variables. A binary outcome of whether a subject had TSC-LAM or sporadic LAM was compared with predictor variables, such as age, time of first symptom, length of follow-up, dyspnoea, haemoptysis, lymphatic system involvement, angiomyolipomas, pulmonary function tests and variables related to cardiopulmonary exercise tests. Variables that resulted in p-values $<0.15$ were used in a multivariate logistic regression model to predict factors that were associated with TSC-LAM. The level of statistical significance was set at $\mathrm{p}<0.05$ for variables to remain in the final model. The odds ratios (OR) and their $95 \%$ confidence intervals were also derived. For comparison of proportions of TSC and TSC-LAM patients with lung function abnormalities we employed the Chi-squared test. The Wilcoxon-Mann-Whitney test was used to compare the proportions of rates of lung function decline between TSC and sporadic LAM patients. Analysis of variance and the Tukey-Kramer procedure were employed to compare initial and final cyst scores for both TSC-LAM and sporadic LAM patients.

All data, except the estimated yearly rate of change in lung function and cyst scores, are shown as mean \pm SD.

\section{Results}

\section{Patient characteristics}

Of 554 patients with LAM, 94 had TSC-LAM and 460 had sporadic LAM (fig. 1). Demographic features of the 94 patients with TSC-LAM are shown in table S1 of the online supplementary material. 61 of the 94 patients were diagnosed with TSC (mean age 18.3 \pm 14.7 years) before being diagnosed with LAM (mean age $36 \pm 10$ years). In 26 patients, the diagnosis of TSC (mean age $41.6 \pm 10.6$ years) was made after the diagnosis of LAM; 14 of the 26 had a lung biopsy. The mean age at the time of the diagnosis of LAM was $37 \pm 10.4$ years. 22 of the 26 patients were diagnosed with TSC at NIH. In seven of the 94 patients, the diagnoses of TSC and LAM were made at the same time (mean age $37.4 \pm 12$ years). The diagnosis of TSC was based on the presence of at least two major criteria or one major and one or more minor features [6].

38 of the 94 patients were brought to NIH under a screening protocol for TSC and were subsequently found to have LAM. Five of these patients had lung biopsies; the remaining patients were diagnosed by CT scan. All but seven patients had respiratory symptoms. The remaining 56 patients were brought in because of cystic lung disease and TSC or cystic lung disease and no prior diagnosis of TSC. 25 of the 56 underwent tissue biopsies and the remaining 31 were diagnosed by CT scan [33]. Seven of the 56 patients had no respiratory symptoms.

Table 1 shows age of first LAM-related symptoms, age at diagnosis and at enrolment in our study, method of diagnosis, mode of presentation and other features, for the 554 patients. In 37 TSC-LAM patients, the diagnosis of LAM was made by tissue biopsy and, in the remaining 57, by the presence of lung cysts and angiomyolipomas in the setting of TSC [33]. Of the 460 sporadic LAM patients, 308 were diagnosed by tissue biopsy and the remaining 152 were diagnosed by clinical and radiological criteria [33].

\section{Pulmonary function studies}

Initial pulmonary function data for TSC-LAM and sporadic LAM patients are shown in figure 2a and in table S2 of the online supplementary material. 31 (33\%) TSC-LAM patients had airflow obstruction

FIGURE 1 Flow diagram showing the numbers of tuberous sclerosis complex (TSC)/lymphangioleiomyomatosis (LAM) and sporadic LAM patients seen initially and those who had pulmonary function tests (PFT) and cardiopulmonary exercise tests (CPET) spanning several years.

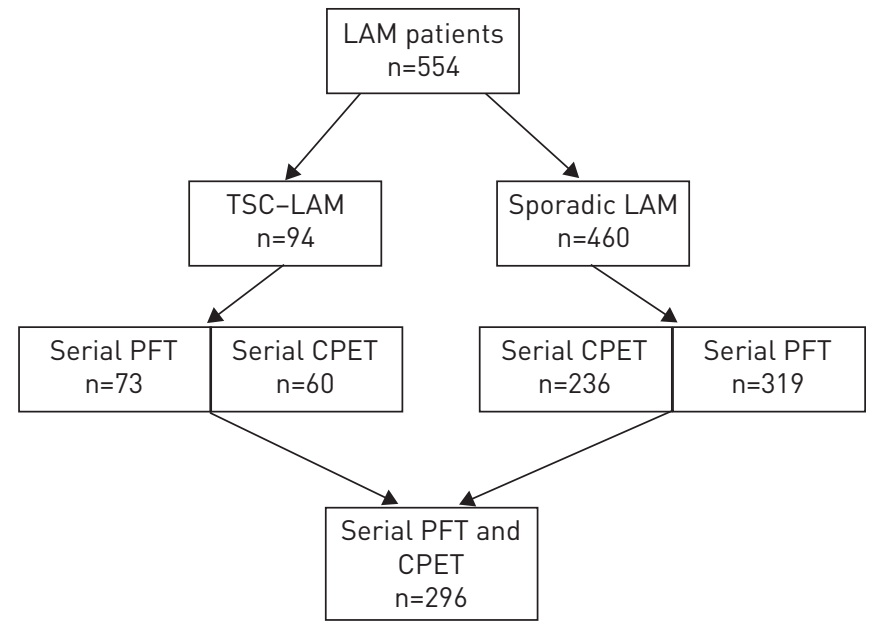


TABLE 1 Demographic features of 554 patients with lymphangioleiomyomatosis (LAM)

\begin{tabular}{lcc} 
Demographics & TSC-LAM & Sporadic LAM \\
\hline Patients $n$ & 94 & 460 \\
White & $79(84)$ & $397(86.3)$ \\
Black & $7(7.4)$ & $26(5.6)$ \\
Hispanic & $5(5.3)$ & $10(2.1)$ \\
Asian & $2(2.1)$ & $25(5.4)$ \\
Other & $1(1.0)$ & $2(0.4)$ \\
Age at LAM diagnosis years & $36.4 \pm 10.4^{*}$ & $41.1 \pm 9.4$ \\
Age at first symptom years & $33.2 \pm 9.9^{*}$ & $37.0 \pm 9.9$ \\
Age at first visit to NIH years & $39.1 \pm 10.2^{*}$ & $43.9 \pm 9.2$ \\
Death during study & $14(14.8)$ & $70(15.2)$ \\
Lung transplantation during study & $4(4.2)$ & $51(11)$ \\
Initial symptoms & & \\
Dyspnoea & $35(37)$ & $222(42)$ \\
Cough & 0 & $19(4)$ \\
Pneumothorax & $38(40)^{*}$ & $13(2.8)$ \\
Haemoptysis & $3(3)$ & $44(10)$ \\
Chylous effusions & $10(11)$ & $33(7)$ \\
Abdominal, pelvic or back pain & $8(9)$ & $59(13)$ \\
$\quad$ No respiratory symptoms & $19(19)$ & $8(2)$ \\
Extrapulmonary findings & & \\
Lymphangioleiomyomas & $12(12.8)$ & $177(38)$ \\
Angiomyolipomas & $90(95.7)$ & $190(41.3)$ \\
Bilateral angiomyolipomas & $80(85.1)$ & $61(13.2)$ \\
Chylous effusions, any time & $13(13.8)$ & $93(20)$ \\
Mode of diagnosis of LAM & & $308(67)$ \\
Tissue biopsy & $37(39)$ & $152(33)$ \\
Clinical data and computed tomography & $57(61)$ & $14(15)$ \\
Bronchodilator response & $107(23)$ & $105(22.8)$ \\
Post-menopausal & $16(17)$ & $207(45)$ \\
Therapies received & $47(51)$ & $41(8.9)$ \\
Progesterone/leuprolide & $8(8.5)$ & \\
Sirolimus & & \\
\hline
\end{tabular}

Data are presented as $n(\%)$ or mean \pm SEM, unless otherwise stated. TSC: tuberous sclerosis complex; NIH: National Institutes of Health. ${ }^{*} p<0.05$ compared with sporadic LAM.

(table 2). Overall, 38 (40\%) out of the 94 patients had abnormal diffusing capacity of the lung for CO (DLCO). 40 (43\%) had normal lung function.

Data for an average follow-up of 5.1 \pm 3 years were available for 73 LAM-TSC and 319 sporadic LAM patients (fig. 1) (initial data given in table S3 of the online supplementary material). Although FEV1 and DLCO were significantly higher in TSC-LAM patients than sporadic LAM patients, no statistically significant differences were observed between the two groups in rates of decline of any of the respiratory variables (fig. 2b). The yearly rates of decline in FEV1 \% predicted for TSC-LAM and sporadic LAM were, respectively, $1.7 \pm 0.4 \%$ predicted $(69.6 \pm 12.2 \mathrm{~mL})$ and $2.2 \pm 0.2 \%$ predicted $(81.5 \pm 6.4 \mathrm{~mL})(\mathrm{p}=0.302)$; the rates of yearly decline in DLCO were respectively, $2.8 \pm 0.6 \%$ predicted $\left(0.78 \pm 0.14 \mathrm{~mL} \cdot \mathrm{min}^{-1} \cdot \mathrm{mmHg}^{-1}\right)$ and $2.1 \pm 3.9 \%$ predicted $\left(0.57 \pm 0.04 \mathrm{~mL} \cdot \mathrm{min}^{-1} \cdot \mathrm{mmHg}^{-1}\right)(\mathrm{p}=0.282)$.

To further study differences between TSC-LAM and sporadic LAM patients, we performed two additional types of analysis. First, we compared the proportions of patients showing airflow obstruction, diffusion impairment or normal lung function and found that greater proportions of sporadic LAM patients had functional abnormalities than TSC-LAM patients and the difference between both groups was statistically significant (table 2).

In addition, we stratified the rates of yearly changes in FEV1 and DLCO for TSC-LAM and sporadic LAM patients and performed a statistical analysis of the data. This analysis showed that the proportion of patients with higher rates of FEV1 decline was significantly greater in sporadic than in TSC-LAM (table 3).

\section{Cardiopulmonary exercise testing}

Results from the initial cardiopulmonary exercise tests (CPET) of 76 TSC-LAM patients and 340 sporadic LAM patients are shown on figure $2 \mathrm{c}$, and in table S4 of the online supplementary material. 18 (23.6\%) of 

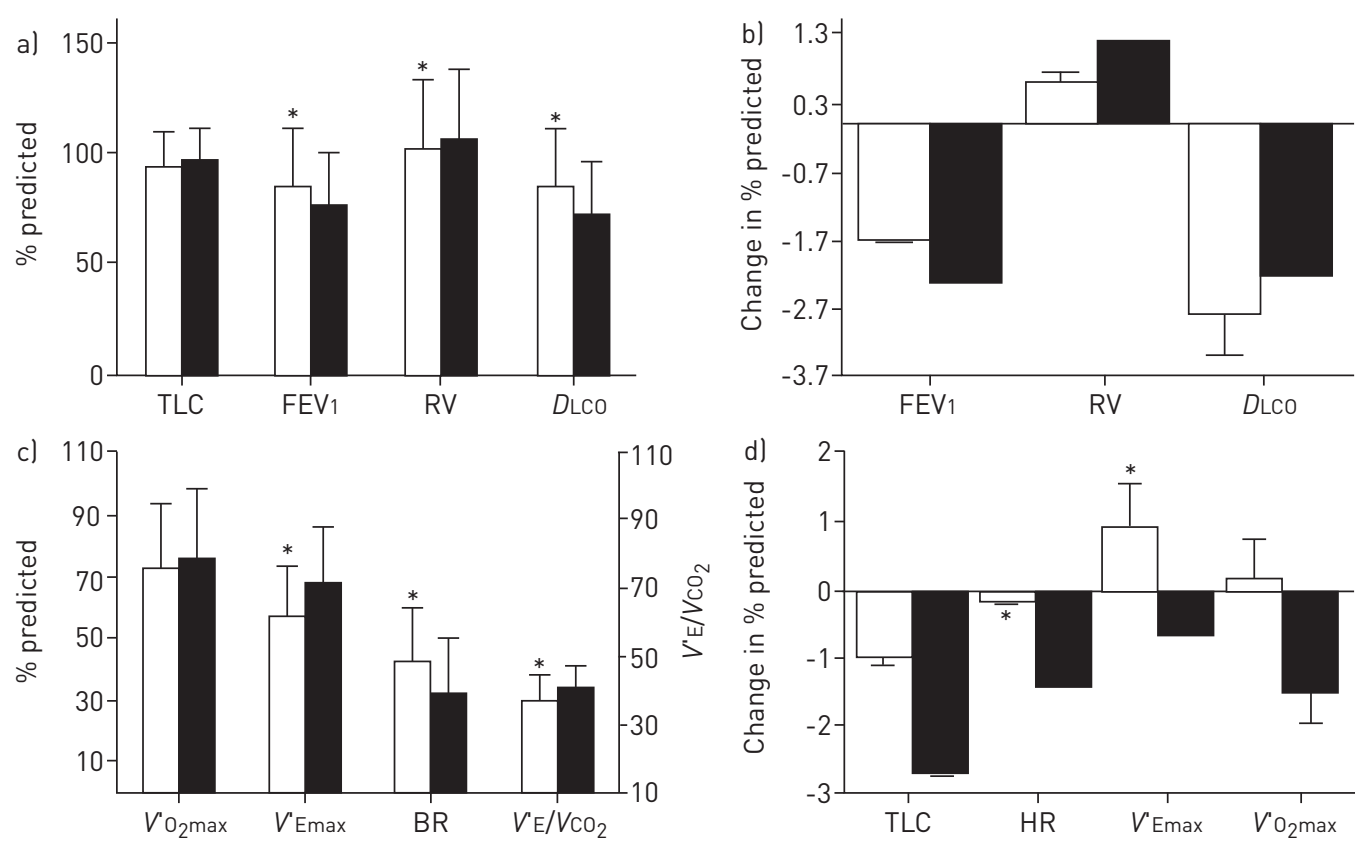

FIGURE 2 a) Pulmonary function data in 94 tuberous sclerosis complex (TSC)/lymphangioleiomyomatosis (LAM) (white bars) and 460 sporadic LAM (black bars) patients obtained at the time of the first visit to the National Institutes of Health (NIH). Overall TSC-LAM patients have significantly higher forced expiratory volume in $1 \mathrm{~s}\left(\mathrm{FEV}_{1}\right)$ and diffusing capacity of the lung for CO (DLCO) and lower residual volume (RV) than sporadic LAM patients. b) Yearly changes in FEV1 \% predicted, RV and DLCO, in 73 patients with TSC-LAM (white bars) and 319 patients with sporadic LAM (black bars) followed for $>5$ years, expressed as mean \pm SEM. No statistically significant differences were observed between TSC-LAM and sporadic LAM patients. c): Initial cardiopulmonary exercise data in 76 TSC-LAM (white bars) and 340 sporadic LAM patients (black bars) obtained at the time of the first visit to NIH. TSC-LAM patients have significantly higher breathing reserve $(\mathrm{BR})$ and lower minute ventilation at peak exercise ( $V^{\prime}$ Emax) and lower ventilatory equivalent for $\mathrm{CO}_{2}$ (minute ventilation divided by $\mathrm{CO}_{2}$ uptake $\left(V^{\prime} \mathrm{E} / V^{\prime} \mathrm{CO}_{2}\right)$ ) than sporadic LAM patients. d) Yearly changes in exercise variables in 60 TSC-LAM (white bars) and 236 sporadic LAM patients (black bars) followed for approximately 4 years, expressed as mean \pm SEM. There was a statistically significant difference in the rates of change in peak heart rate and peak minute ventilation between TSC-LAM and sporadic LAM patients. TLC: total lung capacity; $\mathrm{V}^{\prime} \mathrm{O}_{2}$ max: maximal oxygen uptake; HR: heart rate. *: $\mathrm{p}<0.05$ compared with sporadic LAM patients.

the 76 TSC-LAM patients experienced a drop in oxygen saturation $\left(\mathrm{SaO}_{2}\right) \geq 4 \%$ (mean $\left.7.0 \pm 2.7 \%\right)$. 17 TSCLAM patients failed to reach anaerobic threshold. Seven of these patients had profound hypoxaemia and four had decreased breathing reserve. Analysis of the initial CPET data showed that TSC-LAM patients had greater breathing reserve and lower peak minute ventilation ( $\left.V^{\prime} \mathrm{Emax}\right)$ and ventilatory equivalent for $\mathrm{CO}_{2}$ (minute ventilation divided by $\mathrm{CO}_{2}$ uptake $\left(V^{\prime} \mathrm{E} / V^{\prime} \mathrm{CO}_{2}\right)$ ) at anaerobic threshold than sporadic LAM patients (fig. 2c). There were no other statistically significant differences in exercise variables between TSC-LAM and sporadic LAM patients. Longitudinal CPET data comprising approximately 4 years of followup were available for 60 TSC-LAM and 236 sporadic LAM patients (fig. 2d). We found a greater decrease in peak heart rate $\%$ predicted in sporadic LAM than TSC-LAM patients $(-1.4 \pm 0.2$ versus $-0.14 \pm 0.31 \%$; $\mathrm{p}=0.002)$ and a greater decline in $V^{\prime} \mathrm{Emax} \%$ predicted in sporadic LAM than TSC-LAM patients $(-0.6 \pm 0.4$ versus $0.9 \pm 0.7 \%, \mathrm{p}=0.011$ ).

TABLE 2 Proportions of tuberous sclerosis complex (TSC) and sporadic lymphangioleiomyomatosis (LAM) patients with functional abnormalities

\begin{tabular}{lccc} 
& TSC-LAM & Sporadic LAM & p-value $^{\#}$ \\
\hline Patients n & 94 & 460 & \\
Airflow obstruction & $31(32.9)$ & $234(50.8)$ & 0.002 \\
Diffusion abnormality & $38(40.4)$ & $282(61.3)$ & $<0.001$ \\
Airflow obstruction and diffusion abnormality & $25(26.5)$ & $194(42.1)$ & 0.005 \\
Normal pulmonary function & $40(42.5)$ & $122(26.5)$ & 0.002 \\
\hline Data are presented as n (\%), unless otherwise stated. * : Using a Chi-squared test. & \\
\hline
\end{tabular}


TABLE 3 Proportion of rates of functional decline in lung function in 73 patients with tuberous sclerosis complex (TSC) and sporadic lymphangioleiomyomatosis (LAM) and 319 patients with sporadic LAM ${ }^{\#}$

\begin{tabular}{|c|c|c|c|c|}
\hline & \multicolumn{2}{|c|}{ TSC-LAM } & \multicolumn{2}{|c|}{ Sporadic LAM } \\
\hline & FEV1 & DLco & $\mathrm{FEV}_{1} \pi$ & DLco \\
\hline Patients n & 73 & 73 & 319 & 317 \\
\hline \multicolumn{5}{|l|}{ Decline in \% predicted } \\
\hline$>10 \%$ & $1(1.3)$ & $4(5.4)$ & $11(3.4)$ & $14(4.3)$ \\
\hline $8-10 \%$ & $3(4.1)$ & $2(2.7)$ & $9(2.8)$ & $10(3.1)$ \\
\hline $6-8 \%$ & $5(6.8)$ & $8(10.9)$ & $19(5.9)$ & 12 (3.7) \\
\hline $4-6 \%$ & $2(2.7)$ & $4(5.4)$ & 32 (10.0) & 38 (11.9) \\
\hline $2-4 \%$ & 13 (17.8) & $15(20.5)$ & 87 (27.2) & 84 (26.3) \\
\hline $0-2 \%$ & $31(42.4)$ & 27 (36.9) & 95 (29.7) & $90(28.2)$ \\
\hline
\end{tabular}

Data are presented as $\mathrm{n}(\%)$, unless otherwise stated. FEV1: forced expiratory volume in $1 \mathrm{~s}$; D Lco: diffusing capacity of the lung for $\mathrm{CO}$. " : patients were followed for $5.1 \pm 3$ years; ${ }^{\text {I }} \mathrm{p}=0.048$, using Wilcoxon-MannWhitney test.

Predictors of TSC-LAM and disease severity

A multivariate model was employed to estimate the odds of a patient having TSC-LAM instead of sporadic LAM (table 4). This analysis showed that only the age of diagnosis $(p=0.017)$, age of first respiratory symptoms $(\mathrm{p}=0.007)$ and the presence of angiomyolipomas $(\mathrm{p}=0.003)$ or lymphangioleiomyomas $(\mathrm{p}<0.001)$, were significantly different between patients with TSC-LAM and those with sporadic LAM (table 4). An odds-ratio analysis (table 4) showed that the presence of lymphangioleiomyomas decreased the odds of having TSC-LAM by $74 \%$. The presence of angiomyolipomas increased by 2.5 -times the odds of having TSC-LAM. Finally, for each additional year in age at the time of diagnosis of LAM, the odds of having TSC-LAM decreased by $3.8 \%$ (table 4 ).

Because the frequency of lymphangioleiomyomas was greater in sporadic LAM, we questioned whether this manifestation of extrapulmonary LAM was related to the severity of lung disease. A multivariate analysis showed that patients with lymphangioleiomyomas $(n=143)$ had lower DLCO than patients without lymphangioleiomyomas: $66.0 \pm 23.4 \%$ predicted $\left(13.9 \pm 5.0 \mathrm{~mL} \cdot \mathrm{min}^{-1} \cdot \mathrm{mmHg}^{-1}\right)$ versus $78.6 \pm 25.0 \%$ predicted $\left(16.8 \pm 5.8 \mathrm{~mL} \cdot \mathrm{min}^{-1} \cdot \mathrm{mmHg}^{-1}\right) \quad(\mathrm{p}<0.001)$. A lower DLCO was observed in patients with lymphangioleiomyomas regardless of the co-occurrence of angiomyolipomas. A similar analysis restricted to TSC-LAM patients was not performed because only 12 TSC-LAM patients had lymphangioleiomyomas.

Rate of progression of lung disease in TSC-LAM

We compared changes in cyst scores and lung function of 40 TSC-LAM with 40 age and lung function-matched sporadic LAM patients followed for about six and one half years (table 5). A reading of the first and last imaging studies showed that in 21 TSC-LAM patients the average number of cysts increased from $26 \pm 25$ to $50 \pm 43$. Cyst scores increased from $5.3 \pm 5.9$ to $11.8 \pm 9.6 \%(\mathrm{p}<0.05)$. We further analysed the TSC-LAM CT scan data focusing on 11 young TSC-LAM patients (mean age $=25.7 \pm 3$ years, range $18-30$ ) for

TABLE 4 Multivariate analysis and odds ratio estimates for distinguishing clinical features of tuberous sclerosis complex (TSC) and sporadic lymphangioleiomyomatosis (LAM) and sporadic LAM

\begin{tabular}{lccc} 
& TSC-LAM & Sporadic LAM & p-value \\
\hline Multivariate analysis & & & \\
Patients $\mathrm{n}$ & 73 & 319 & 0.017 \\
Age at diagnosis years & $38.0 \pm 10.7$ & $41.5 \pm 9.2$ & 0.007 \\
Age at first symptoms years & $34.2 \pm 10.3$ & $38.0 \pm 9.5$ & 0.003 \\
Angiomyolipoma & 89 & 36 & $<0.001$ \\
Lymphangioleiomyoma & 15 & 41 & 0.017 \\
Odds ratio estimates & & $2.54(1.372-4.701)$ & 0.003 \\
Angiomyolipomas & & $0.237(0.101-0.554)$ & $<0.001$ \\
Lymphangioleiomyomas & $0.962(0.931-0.993)$ & \\
Age & & \\
\hline
\end{tabular}

Data are presented as mean \pm SEM, $\%$ or OR $(95 \% \mathrm{CI})$, unless otherwise stated. 
TABLE 5 Demographic, physiological and radiological findings in 40 patients with tuberous sclerosis complex (TSC) and sporadic lymphangioleiomyomatosis (LAM) and 40 patients with sporadic LAM

\begin{tabular}{|c|c|c|}
\hline Demographics & TSC-LAM & Sporadic LAM \\
\hline Age years & $40.1 \pm 9.9$ & $39.9 \pm 8.1$ \\
\hline Follow-up time years & $6.5 \pm 2.7$ & $6.5 \pm 3.2$ \\
\hline Deaths & 2 (5) & $3(7.5)$ \\
\hline \multicolumn{3}{|l|}{ Lung function } \\
\hline FEV $1 \mathrm{~L}$ & $2.6 \pm 0.5$ & $2.6 \pm 0.5$ \\
\hline FEV $1 \%$ predicted & $96.8 \pm 18.9$ & $93.8 \pm 15.9$ \\
\hline$D \mathrm{Lco} \mathrm{mL} \cdot \mathrm{min}^{-1} \cdot \mathrm{mmHg}^{-1}$ & $20.4 \pm 3.9$ & $20.5 \pm 4.2$ \\
\hline D Lco $\%$ predicted & $95.4 \pm 19.1$ & $94.8 \pm 16.7$ \\
\hline Decline in $\mathrm{FEV}_{1} \%$ predicted & $1.9 \pm 2.7$ & $1.9 \pm 1.9$ \\
\hline Decline in DLco \% predicted & $2.1 \pm 2.8$ & $1.9 \pm 2.7$ \\
\hline \multicolumn{3}{|l|}{ Cardiopulmonary exercise testing } \\
\hline Work rate \% predicted & $99 \pm 25$ & $109 \pm 24$ \\
\hline Peak heart rate $\%$ predicted & $89 \pm 8$ & $91 \pm 8$ \\
\hline$V^{\prime}$ Emax \% predicted & $57 \pm 16$ & $62 \pm 16$ \\
\hline Breathing reserve $\%$ & $44 \pm 16$ & $38 \pm 20$ \\
\hline$V^{\prime} \mathrm{O}_{2} \max \%$ predicted & $80 \pm 15$ & $86 \pm 21$ \\
\hline$V^{\prime} \mathrm{E} / \mathrm{V}^{\prime} \mathrm{CO}_{2}$ at anaerobic threshold & $32 \pm 7$ & $34 \pm 6$ \\
\hline \multicolumn{3}{|l|}{ Volume occupied by cysts } \\
\hline Initial cyst scores \% & $5.3 \pm 5.9$ & $5.6 \pm 5.2$ \\
\hline Final cyst scores \% & $11.8 \pm 9.6^{\#}$ & $15.3 \pm 9.8^{\#}$ \\
\hline Yearly change in cyst scores $\%$ & $1.0 \pm 1.3$ & $1.4 \pm 1.7$ \\
\hline \multicolumn{3}{|c|}{$\begin{array}{l}\text { Data are presented as mean } \pm \text { SEM or } \mathrm{n}(\%) \text {. \#: Statistically significant from initial cyst score (by ANOVA). } \\
\text { FEV1: forced expiratory volume in the first second; } D L C O \text { : diffusion capacity for carbon monoxide; } V^{\prime} E m a x: \\
\text { minute ventilation at peak exercise; } V^{\prime} \mathrm{O}_{2} \text { max: peak oxygen uptake; } V^{\prime} E / V^{\prime} \mathrm{CO}_{2} \text { : ventilatory equivalent for } \mathrm{CO}_{2} \text {. }\end{array}$} \\
\hline
\end{tabular}

whom we had data spanning $4.3 \pm 3.8$ years. Yearly rates of decline in FEV1 and DLCO were $4.1 \pm 3.6$ and $4.3 \pm 3.8 \%$ predicted, respectively. Visual review of CT scans showed progression of disease from minimal or mild to moderate-to-severe. In six patients, there was quick progression to severe disease. Yearly rates of decline in in FEV1 and DLCO were $5.9 \pm 3.0$ and $5.4 \pm 3.6 \%$ predicted and the percentage of lung volume occupied by cysts increased from $8.1 \pm 6.9$ to $27.6 \pm 5.8 \%$. CT scans of two of these patients are shown in figure 3 .

In 18 out of the 40 sporadic LAM patients, over the period of observation, the average number of cysts increased from $66 \pm 40$ to $94 \pm 60$. Cyst scores increased from $5.6 \pm 5.2$ to $15.3 \pm 9.8 \%(\mathrm{p}<0.05)$ (table 5). There was no significant difference between TSC-LAM and sporadic LAM patients in both initial and final cyst scores. There was also no significant difference in the rate of change in cyst scores for TSC-LAM and sporadic LAM patients: $1.0 \pm 1.3$ versus $1.4 \pm 1.7 \%$; $\mathrm{p}=0.213$ (table 5). Table S5 and figure S1 in the online supplementary material show changes in cyst scores for each TSC-LAM and sporadic LAM patient.

\section{Discussion}

Our study shows that, compared with sporadic LAM patients, TSC-LAM patients were diagnosed with LAM at an earlier age and were more likely to have fewer respiratory symptoms. The presence of angiomyolipomas increased the odds of a LAM patient having TSC-LAM. Conversely, the presence of lymphangioleiomyomas decreased the odds of a patient having TSC-LAM. A greater proportion of TSCLAM patients presented with normal lung function than sporadic LAM patients. Furthermore, although there was no statistically significant difference in FEV1 and DLCO rates of decline between TSC-LAM and sporadic LAM patients, stratification of rates of functional decline according to grade showed that more TSC-LAM patients had lesser rates of FEV1 decline than sporadic LAM patients.

These differences in disease severity at the time of presentation and subsequent functional decline could be explained by recruitment bias. Indeed, 38 TSC-LAM patients were referred to NIH under a screening protocol and were only later found to have LAM. It is likely that such a cohort would have milder lung disease than a population of TSC-LAM patients presenting with respiratory symptoms. This referral bias may have resulted in more TSC-LAM patients having milder lung disease than sporadic LAM patients. In agreement, we found that patients diagnosed with LAM after being diagnosed with TSC tended to have higher FEV1 ( $83 \pm 27$ versus $75 \pm 26 \%$ predicted) and DLCO ( $82 \pm 29$ versus $77 \pm 23 \%$ predicted) at the time of 

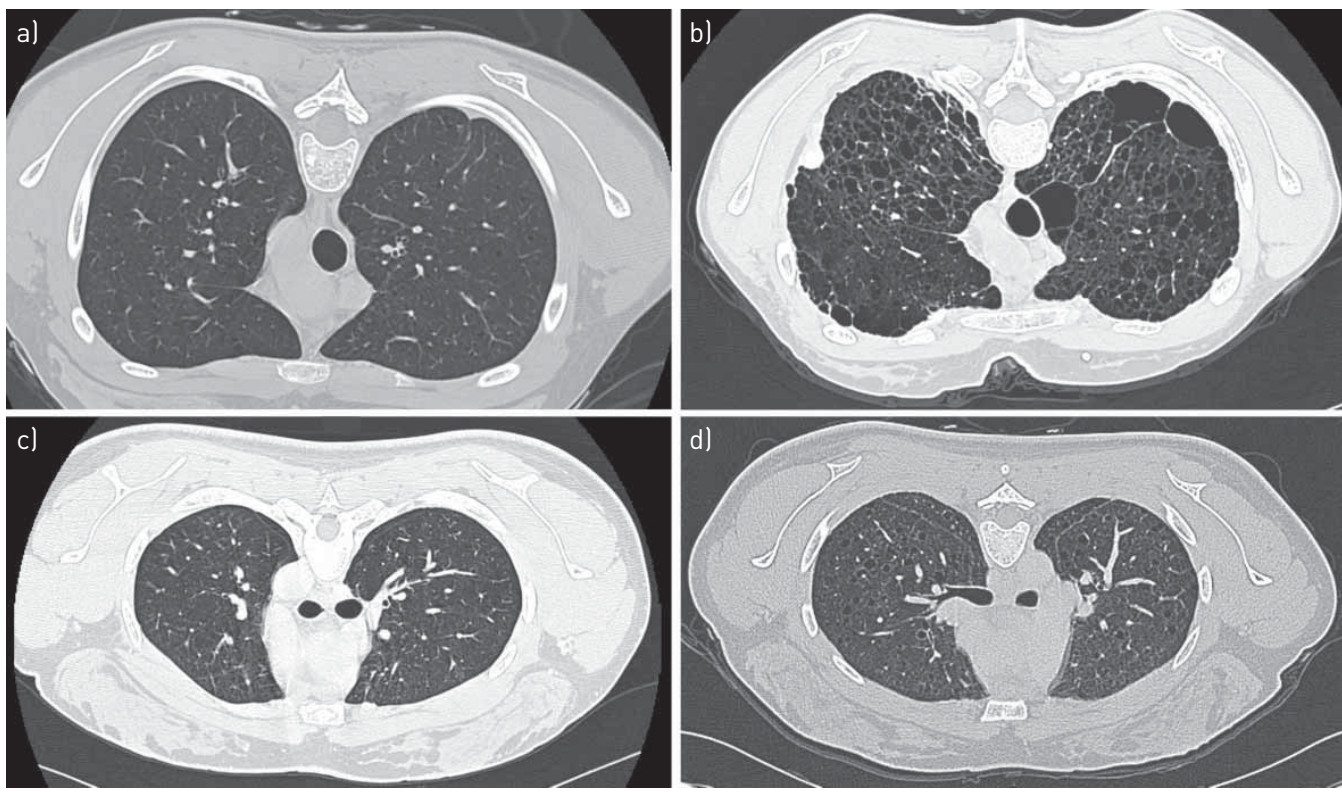

FIGURE 3 a) Lung computed tomography scan of a 19-year-old woman with tuberous sclerosis complex (TSC)/ lymphangioleiomyomatosis (LAM) showing minimal cystic changes. b) 5 years later, the computed tomography scan shows severe disease. c). Lung computed tomography scan of a 25 -year-old woman with TSC-LAM showing minimal cystic changes. d) 5 years later, the computed tomography scan shows more severe disease.

the first NIH visit than patients in whom the diagnosis of LAM preceded the diagnosis of TSC, although the difference was not statistically significant.

The finding that a relatively large number of TSC patients presenting with LAM only belatedly were diagnosed with TSC, is consistent with data from a prior study showing that $45(59 \%)$ of a total 79 TSC patients were only diagnosed with TSC in adulthood. 30 (66\%) of the 45 patients reported clinical features consistent with TSC that antedated the eventual diagnosis of TSC by a median time of 21.5 years [33]. In summary, our TSC-LAM population consisted of a mix of patients presenting as LAM and another presenting as TSC. We propose that the make-up of our cohort corresponds to the most frequent presentation of TSC patients, either as TSC or LAM.

A lower frequency of lymphatic involvement observed in TSC-LAM patients could have accounted for their lesser severity of lung disease. We found that patients with lymphangioleiomyomas had a lower DLCO than patients with less lymphatic involvement and our study showed that lymphangioleiomyomas are more prevalent in sporadic LAM. Lymphatic disease in LAM is known to be associated with increased blood levels of vascular endothelium growth factor D (VEGF-D), and data from the MILES (The Multicenter International LAM Efficacy of Sirolimus) trial have shown that increased levels of VEGF-D are associated with more severe lung function abnormalities, less exercise tolerance and greater oxygen requirements [34]. It is possible that lymphatic involvement with LAM cell infiltration may obstruct lymphatic flow from the lungs causing impairment of gas exchange, which is reflected by diminished DLCO and hypoxaemia. This hypothesis is consistent with reports that lymphangioleiomyomas and pulmonary infiltrates resolve after treatment with sirolimus, resulting in improvement in lung function [35-37]. However, there are no data regarding the potential value of measurement of serum VEGF-D levels in predicting the prognosis of a woman with TSC who has been diagnosed with LAM. Of the available methods of assessing the prognosis of LAM, measuring the rates of decline in lung function over time and quantitatively grading CT scans remain the most practical and useful tools.

Initial cardiopulmonary exercise testing data are consistent with the differences in degree and frequency of pulmonary function abnormalities in TSC-LAM and sporadic LAM patients. Breathing reserve was higher in TSC-LAM than sporadic LAM patients. Peak $V^{\prime} E \max$ and $V^{\prime} \mathrm{E} / V^{\prime} \mathrm{CO}_{2}$ at anaerobic threshold were higher in sporadic LAM reflecting a greater ventilatory response to exercise. Because TSC-LAM patients had better lung function, their ventilatory response to exercise was less, resulting in a lower $V^{\prime} \mathrm{E} / V^{\prime} \mathrm{CO}_{2}$, lower $V^{\prime}$ Emax and greater breathing reserve.

One of the questions that we wanted to address in this study was whether the detection of a few lung cysts on a CT scan of women with TSC equates to the presence of LAM and whether such patients eventually develop clinically significant lung disease, their course is similar to that seen in sporadic LAM. We found 
that the presence of a few lung cysts and normal lung function in a woman with TSC does not necessarily predict a good outcome. In fact, we identified 11 young TSC-LAM patients with few lung cysts and normal lung function who rapidly progressed to moderate-to- severe lung disease, reflected by high rates of lung function decline and increased percentage of lung volume occupied by cysts. These findings suggest that, as in sporadic LAM, lung disease in TSC-LAM patients may worsen rapidly with age and run a course similar to sporadic LAM.

Our data are consistent with the results of a study involving women with TSC, showing that the risk of LAM was age-dependent, increasing from $27 \%$ in subjects aged $<21$ years, to $81 \%$ in subjects aged $>40$ years [25]. A European Respiratory Society consensus statement recommended screening of TSC patients by CT scans at 18 years of age and repeating the study when 30 years old [33]. A more recent statement regarding surveillance of women with TSC and demonstrable lung cysts also recommended CT scans every 2-3 years and annual pulmonary function tests [38]. In our study, we report longitudinal physiological and CT data showing that, although TSC-LAM patients, as a group, tended to have milder and less progressive lung disease than sporadic LAM patients, individual patients, specifically women aged 18-30 years, may experience rapid declines in lung function. Any approach other than close monitoring of respiratory symptoms, lung function and exercise tolerance, along with imaging studies, in TSC women in that age group, would fail to detect the presence of clinically significant cystic lung disease before the age of 30 years. Thus, close medical follow-up of all women with TSC and lung cysts, including pulmonary function tests and computed tomography, is recommended. This approach is justified by evidence that treatment of LAM with sirolimus is effective in slowing progression of lung disease [37, 39].

\section{References}

1 Ryu JH, Moss J, Beck GJ, et al. The NHLBI lymphangioleiomyomatosis registry: characteristics of 230 patients at enrollment. Am J Respir Crit Care Med 2006; 173: 105-111.

2 McCormack FX. Lymphangioleiomyomatosis: a clinical update. Chest 2008; 133: 507-516.

3 Meraj R, Wikenheiser-Brokamp KA, Young LR, et al. Lymphangioleiomyomatosis: new concepts in pathogenesis, diagnosis, and treatment. Semin Respir Crit Care Med 2012; 33: 486-497.

4 Ferrans VJ, Yu ZX, Nelson WK, et al. Lymphangioleiomyomatosis (LAM): a review of clinical and morphological features. J Nippon Med Sch 2000; 67: 311-329.

5 Yu J, Astrinidis A, Henske EP. Chromosome 16 loss of heterozygosity in tuberous sclerosis and sporadic lymphangiomyomatosis. Am J Respir Crit Care Med 2001; 164: 1537-1540.

$6 \quad$ Curatolo P, Bombardieri R, Jozwiak S. Tuberous sclerosis. Lancet 2008; 372: 657-668.

7 Harknett EC, Chang WY, Byrnes S, et al. Use of variability in national and regional data to estimate the prevalence of lymphangioleiomyomatosis. Q J Med 2011; 104: 971-979.

8 Smolarek TA, Wessner LL, McCormack FX, et al. Evidence that lymphangiomyomatosis is caused by TSC2 mutations: chromosome $16 \mathrm{p} 13$ loss of heterozygosity in angiomyolipomas and lymph nodes from women with lymphangiomyomatosis. Am J Hum Genet 1998; 62: 810-815.

9 Carsillo T, Astrinidis A, Henske EP. Mutations in the tuberous sclerosis complex gene TSC2 are a cause of sporadic pulmonary lymphangioleiomyomatosis. Proc Natl Acad Sci USA 2000; 97: 6085-6090.

10 Knudson AG. Hereditary cancer: two hits revisited. J Cancer Res Clin Oncol 1996; 122: 135-140.

11 Crooks DM, Pacheco-Rodriguez G, DeCastro RM, et al. Molecular and genetic analysis of disseminated neoplastic cells in lymphangioleiomyomatosis. Proc Natl Acad Sci USA 2004; 101: 17462-17467.

12 Cai X, Pacheco-Rodriguez G, Fan QY, et al. Phenotypic characterization of disseminated cells with TSC2 loss of heterozygosity in patients with lymphangioleiomyomatosis. Am J Respir Crit Care Med 2010; 182: 1410-1418.

13 Bittmann I, Rolf B, Amann G, Löhrs U. Recurrence of lymphangioleiomyomatosis after single lung transplantation, new insights into pathogenesis. Human Pathol 2003; 34: 95-98.

14 Karbowniczek M, Astrinidis A, Balsara BR, et al. Recurrent lymphangiomyomatosis after transplantation: genetic analyses reveal a metastatic mechanism. Am J Respir Crit Care Med 2003; 167: 976-982.

15 Hayashi T, Kumasaka T, Mitani K, et al. Prevalence of uterine and adnexal involvement in pulmonary lymphangioleiomyomatosis: a clinicopathologic study of 10 patients. Am J Surg Pathol 2011; 35: 1776-1285.

16 Corrin B, Liebow AA, Friedman PJ. Pulmonary lymphangioleiomyomatosis: a review. Am J Pathol 1975; 79: 347-382.

17 Dwyer JM, Hickie JB, Garvan J. Pulmonary tuberous sclerosis. Report of three patients and a review of the literature. Q J Med 1971; 40: 115-125.

18 Shepherd CW, Gomez MR, Lie JT, et al. Causes of death in tuberous sclerosis. Mayo Clin Proc 1971; 66: 792-796.

19 Castro M, Shepherd CW, Gomez MR, et al. Pulmonary tuberous sclerosis. Chest 1995; 107: 189-195.

20 Costello LC, Hartman TE, Ryu JH. High frequency of pulmonary lymphangioleiomyomatosis in women with tuberous sclerosis complex. Mayo Clin Proc 2000; 75: 591-594.

21 Moss J, Avila N, Barnes PM, et al. Prevalence and clinical characteristics of lymphangioleiomyomatosis (LAM) in patients with tuberous sclerosis complex. Am J Respir Crit Care Med 2001; 163: 669-671.

22 Franz DN, Brody A, Meyer C, et al. Mutational and radiographic analysis of pulmonary disease consistent with lymphangioleiomyomatosis and micronodular pneumocyte hyperplasia in women with tuberous sclerosis. Am J Respir Crit Care Med 2001; 164: 661-668.

23 Muzykewicz DA, Sharma A, Muse V, et al. TSC1 and TSC2 mutations in patients with lymphangioleiomyomatosis and tuberous sclerosis complex. J Med Genet 2009; 46: 465-468.

24 Adriaensen ME, Schaefer-Prokop CM, Duyndam DA, et al. Radiological evidence of lymphangioleiomyomatosis in female and male patients with tuberous sclerosis complex. Clin Radiol 2011; 66: 625-628.

25 Cudzilo CJ, Szczesniak RD, Brody AS, et al. Lymphangioleiomyomatosis screening in women with tuberous sclerosis. Chest 2013; 144: 578-585. 
Taveira-DaSilva AM, Stylianou MP, Hedin CJ, et al. Decline in lung function in patients with lymphangioleiomyomatosis treated with or without progesterone. Chest 2004; 126: 1867-1874.

27 Avila NA, Dwyer AJ, Rabel A, et al. Sporadic lymphangioleiomyomatosis and tuberous sclerosis complex with lymphangioleiomyomatosis: comparison of CT features. Radiology 2007; 242: 277-285.

28 Yao J, Taveira-DaSilva AM, Colby TV, et al. CT grading of lung disease in lymphangioleiomyomatosis. AJR Am J Roentgenol 2012; 199: 787-793

29 Seibert D, Hong CH, Takeuchi F, et al. Recognition of tuberous sclerosis in adult women: delayed presentation with life-threatening consequences. Ann Intern Med 2011; 154: 806-813.

30 Miller MR, Hankinson J, Brusasco V, et al. Standardisation of spirometry. Eur Respir J 2005; 26: 319-338

31 Macintyre N, Crapo RO, Viegi G, et al. Standardisation of the single-breath determination of carbon monoxide uptake in the lung. Eur Respir J 2005; 26: 720-725.

32 Taveira-DaSilva AM, Stylianou MP, Hedin CJ, et al. Maximal oxygen uptake and severity of disease in lymphangioleiomyomatosis. Am J Respir Crit Care Med 2003; 168: 1427-1431.

33 Johnson SR, Cordier JF, Lazor R, et al. European Respiratory Society guidelines for the diagnosis and management of lymphangioleiomyomatosis. Eur Respir J 2010; 35: 14-26.

34 Young L, Lee HS, Inoue Y, et al. Serum VEGF-D a concentration as a biomarker of lymphangioleiomyomatosis severity and treatment response: a prospective analysis of the Multicenter International Lymphangioleiomyomatosis Efficacy of Sirolimus (MILES) trial. Lancet Respir Med. 2013; 1: 445-452.

35 Taveira-DaSilva AM, Hathaway O, Stylianou M, et al. Changes in lung function and chylous effusions in patients with lymphangioleiomyomatosis treated with sirolimus. Ann Int Med 2011; 154: 797-805.

36 Moua T, Olson EJ, Jean HC, et al. Resolution of chylous pulmonary congestion and respiratory failure in lymphangioleiomyomatosis with sirolimus therapy. Am J Respir Crit Care Med 2012; 186: 389-390.

37 Cottin V. Treatment of lymphangioleiomyomatosis: building evidence in orphan diseases. Eur Respir J 2014; 43: 966-969.

38 Krueger DA, Northrup H; International Tuberous Sclerosis Complex Consensus Group. Tuberous sclerosis complex diagnostic criteria update: recommendations of the 2012 international tuberous sclerosis complex consensus conference. Pediatr Neurol 2013; 49: 243-254.

39 McCormack FX, Inoue Y, Moss J, et al. National Institutes of Health Rare Lung Diseases Consortium; MILES Trial Group. Efficacy and safety of sirolimus in lymphangioleiomyomatosis. N Engl J Med 2011; 364: $1595-606$. 